\title{
Retrieval of the two-dimensional slope field by the SWIM spectrometer of the CFOSAT satellite: discussion of the algorithm
}

\author{
V. Yu. Karaev ${ }^{1}$, M. A. Panfilova ${ }^{1}$, M. S. Ryabkova ${ }^{1}$, Yu. A. Titchenko ${ }^{1}$, E. M. Meshkov ${ }^{1}$, and X. Li ${ }^{2}$ \\ Received 2 November 2021; accepted 2 December 2021; published 23 December 2021.
}

In October 2018, the Chinese-French satellite CFOSAT was launched into low-earth orbit, which carries the French SWIM spectrometer and the Chinese scatterometer RFSCAT. The SWIM (Ku-band) spectrometer measures the normalized radar cross section (NRCS) at small incidence angles $\left(0^{\circ}-11^{\circ}\right)$ and for the first time measurements are performed at different azimuthal angles in a size-limited area where sea waves can be considered homogeneous. At small incidence angles, the backscattering mechanism is quasi-specular and the reflected field is calculated in the Kirchhoff approximation. Due to this, it becomes possible to retrieve the parameters of large-scale, in comparison with the length of the electromagnetic wave, sea waves (hereinafter referred to as large-scale waves). It has been shown that the common approach to measuring the mean square slopes ( $\mathrm{mss}$ ) of large-scale waves leads to large errors. For the measurement scheme at three azimuthal angles, an algorithm was developed for determining the direction of propagation of the dominant wave system, the mss of large-scale waves along the sounding direction and the unnormalized correlation coefficient between the slopes of large-scale waves along the $X$ and $Y$ axes (hereinafter referred to as the correlation coefficient), which requires solving a system of transcendental equations. If we neglect the correlation coefficient in comparison with the mss of large-scale waves, then the solution is in an analytical form. The results of the simplified algorithm are compared with the exact solution. The values of the mss of large-scale waves obtained with the simplified approach can be used both to estimate the intensity of sea waves and as the initial conditions for solving the transcendental equation. The developed algorithms will be used to process the data of the SWIM spectrometer, which will allow for the first time to retrieval a two-dimensional field of large-scale wave slopes. KEYWORDS: Mean square slopes of large-scale waves; normalized radar cross section; small incidence angles; spectrometer SWIM; retrieval algorithms.

Citation: Karaev, V. Yu., M. A. Panfilova, M. S. Ryabkova, Yu. A. Titchenko, E. M. Meshkov, and X. Li (2021), Retrieval of the two-dimensional slope field by the SWIM spectrometer of the CFOSAT satellite: discussion of the algorithm, Russ. J. Earth. Sci., 21, ES6004, doi:10.2205/2021ES000784.

\section{Introduction}

\footnotetext{
${ }^{1}$ Institute of Applied Physics RAS, Nihzny Novgorod, Russia

${ }^{2}$ School of Marine Sciences, Nanjing University of Information Science and Technology, Nanjing, China
}

Copyright 2021 by the Geophysical Center RAS. http://rjes.wdcb.ru/doi/2021ES000784-res.html
The oceans have a significant impact on the Earth's climate; therefore, remote sensing facilities are constantly being improved. During the interaction of the atmosphere and the ocean, processes of heat and gas exchange take place, and for the transition from qualitative estimates to quantita- 
tive values, information on the near-surface wind speed and parameters of sea waves is needed.

Altimeters measure the significant wave height $(\mathrm{SWH})$ and the data are successfully assimilated into numerical wave models [Birol et al., 2002 Martina et al., 2015, increasing the accuracy of wave climate predictions. Another important characteristic of the sea surface is mean square slopes (mss).

At small incidence angles the reflected radar signal contains information on the parameters of large-scale waves, and the first algorithms for determining the parameters of large-scale waves appeared in the 70s, for example, [Garnakeryan and Sosunov, 1978. However, the developed algorithms have not found practical application, since in experiments, it is rather difficult to organize measurements at small incidence angles. In the course of further studies of the backscattering of microwaves by the sea surface, the main attention was attracted to measurements at middle incidence angles.

The appearance in 1997 of an orbital Ku-band precipitation radar installed on the TRMM (Tropical Rainfall Measuring Mission) satellite [TRMM, 2001 and performing measurements at small incidence angles aroused interest in the problem of measuring the $\mathrm{mss}$ of large-scale waves, for example, [Chu et al., 2012a, 2012b: Freilich and Vanhoff, 2003, Karaev et al., 2012, 2019, Nouguier et al., 2016; Tran et al., 2007. By analogy with the optical measurements by Cox and Munk 1954], the coefficients of the Gramm-Charlier series were calculated to describe the distribution function of the slopes of large-scale waves [Chu et al., 2012b; Chen et al., 2017. Measurements of the mss in slicks were carried out [Panfilova et al., 2018]. It should be noted that the problem of the $\mathrm{mss}$ of largescale waves attracts the attention of researchers and a number of works devoted to this problem can be distinguished, for example, [Danilytchev et al., 2009, Pokazeev and Zapevalov, 2019, Zapevalov, 2020 .

Further development of the study of backscattering at small incidence angles was obtained after the launch in 2014 of the GPM (Global Precipitation Measurement) satellite with a Dual-frequency Precipitation Radar [ $G P M$, 2014, which performs measurements in the $\mathrm{Ku}$ - and Ka-bands, into orbit. However, a significant disadvantage of the precip- itation radar measurement scheme is that the dependence of the NRCS on the incidence angle is measured only at one azimuthal angle, i.e. it is possible to retrieve the $m s s$ of large-scale wave only along the sounding direction.

Retrieval of the 2D slope field requires measurements at different azimuthal angles [Karaev et al., 2006. Such measurements are planned to be performed during an experiment on the Russian segment of the International Space Station [Karaev et al., 2018.

At the end of 2018, the Chinese-French satellite CFOSAT was launched into orbit, on which the Chinese RFSCAT scatterometer and the French SWIM spectrometer (Ku-band) are located. The SWIM spectrometer performs measurements at incidence angles less than $11^{\circ}$ at different azimuthal angles, therefore, for the first time, it becomes possible to retrieve the two-dimensional field of sea surface slopes using the orbital radar data.

This paper discusses the adaptation of the mss retrieval algorithm for the SWIM spectrometer.

\section{Microwave Scattering Model}

The model of backscattering of microwave electromagnetic radiation by the sea surface at small incidence angles in the Kirchhoff approximation acquired its final form in the 70s [Bass and Fuks, 1972. Barrick, 1968. Isakovich, 1952, Valenzuela, 1978 .

The possibility of checking the scattering model appeared after the TRMM satellite was launched into orbit with a Ku-band precipitation radar on board. In well-known works, for example, [Nouguier et al., 2014, 2016. Tran et al., 2007, Yan et al., 2019 , to develop an algorithm for determining the slopes of the sea surface, the authors used the assumption that sea waves are isotropic with Gaussian distribution function of heights. The formula they used for the NRCS is following

$$
\sigma_{0}(\theta)=\frac{\left|R_{\text {eff }}(0)\right|^{2}}{m s s_{0} \cos ^{4} \theta} \exp \left[-\frac{\operatorname{tg}^{2} \theta}{m s s_{0}}\right],
$$

where $m s s_{0}$ is mean square slopes of large-scale waves for isotropic sea waves, $\theta$ - incidence angle. $R_{\text {eff }}$ is the effective reflection coefficient which introduced instead of Fresnel coefficient to take into account the effect of ripple on the amplitude of the reflected signal [Bass and Fuks, 1972. Valenzuela, 1978 . 
The scattering model was further developed by taking into account the curvature of the scattering surface [Boisot et al., 2015]. However, research has shown that the Kirchhoff method as originally formulated better matches measurements of precipitation radar [Yan et al., 2019].

The assumption that the waves are isotropic, used by the authors, does not correspond to reality and using of this approach can be explained only by the fact that measurements were available only at one azimuthal angle. The assumption that the sea waves were isotropic is the only way to "solve" the problem and declare that the mss of large-scale waves is being measured.

In the general case, taking into account the anisotropy of the sea waves, the formula for the NRCS takes the following form [Bass and Fuks, 1972

$$
\begin{gathered}
\sigma_{0}(\theta)=\frac{\left|R_{\mathrm{eff}}(0)\right|^{2}}{2 \cos ^{4} \theta \sqrt{m s s_{x x} m s s_{y y}-m s s_{x y}^{2}}} \times \\
\exp \left[-\frac{\operatorname{tg}^{2} \theta}{2\left(m s s_{x x} m s s_{y y}-m s s_{x y}^{2}\right)} \cdot m s s_{y y}\right],
\end{gathered}
$$

where $m s s_{x x}$ and $m s s_{y y}$ are mean square slopes of large-scale waves along axis $X$ and $Y$ correspondingly; $m s s_{x y}$ is an unnormalized correlation coefficient between slopes along the axes $X$ and $Y$ (further the correlation coefficient). Thus, 4 parameters are unknown: the mss of large-scale waves, the correlation coefficient, and the angle between the sounding direction and the direction of wave propagation.

When analyzing the dependence of NRCS on the incidence angle, two approaches are usually used [Panfilova et al., 2020a]. To illustrate the method, we present the formulas of the two-point algorithm. It uses measurements of the NRCS at two incidence angles and the formula for the NRCS at zero incidence angle becomes:

$$
\sigma_{0}(0)=\sigma_{0}(\theta) \cdot \cos ^{4} \theta \cdot \exp \left(\tan ^{2} \theta \cdot b\right),
$$

where coefficient $b$ is calculated by the following formula

$$
b=\frac{\ln \left(\frac{\sigma_{0}\left(\theta_{1}\right) \cdot \cos ^{4} \theta_{1}}{\sigma_{0}\left(\theta_{2}\right) \cdot \cos ^{4} \theta_{2}}\right)}{\tan ^{2} \theta_{2}-\tan ^{2} \theta_{1}} .
$$

On the other hand, the coefficient $b$ is

$$
b=\frac{m s s_{y y}}{2\left(m s s_{x x} m s s_{y y}-m s s_{x y}^{2}\right)}
$$

or

$$
b=\frac{1}{m s s_{x x}}
$$

for formula (2) and formula (1), respectively.

Before proceeding to the comparison of the formulas, let us consider the azimuthal dependences of the statistical parameters of sea waves.

\section{Sea Waves Parameters}

For further numerical estimates, we will use the wave spectrum model proposed by Ryabkova [Ryabkova et al., 2019. By definition, the $m s s_{x x}$ of largescale waves and the correlation coefficient are calculated by the following formulas

$$
\begin{gathered}
m s s_{x x}= \\
\int_{-\pi}^{\pi} \int_{0}^{\kappa_{c}} \kappa^{2} \cos ^{2} \varphi \cdot S\left(\kappa, \varphi_{0}-\varphi\right) \kappa d \kappa d \varphi \\
m s s_{y y}= \\
\int_{-\pi}^{\pi} \int_{0}^{\kappa_{c}} \kappa^{2} \sin ^{2} \varphi \cdot S\left(\kappa, \varphi_{0}-\varphi\right) \kappa d \kappa d \varphi, \\
\int_{-\pi}^{\pi} \int_{0}^{\kappa_{c}} \kappa^{2} \cos \varphi \sin \varphi \cdot S\left(\kappa, \varphi_{0}-\varphi\right) \kappa d \kappa d \varphi, \\
\int_{0}^{\kappa_{c}} \kappa^{2} S(\kappa) \kappa d \kappa=m s s_{\text {total }}+m s s_{y y},
\end{gathered}
$$

where $S(\kappa, \varphi)$ is the spatial-angular wave spectrum; $\varphi_{0}$ - direction of wave propagation; $m s s_{\text {total }}$ - total mean square slopes of large-scale waves. In the framework of the two-scale model of the sea surface, a boundary wave number $\kappa_{c}$ is introduced, which divides the wave spectrum into large-scale and small-scale components. The formula for the boundary wave number is given in the following paper [Ryabkova et al., 2019].

For numerical estimates, let choose three values of the wind speed: $4 \mathrm{~m} / \mathrm{s}, 8 \mathrm{~m} / \mathrm{s}$, and $12 \mathrm{~m} / \mathrm{s}$ and consider the case of wind waves, i.e. without swell. 


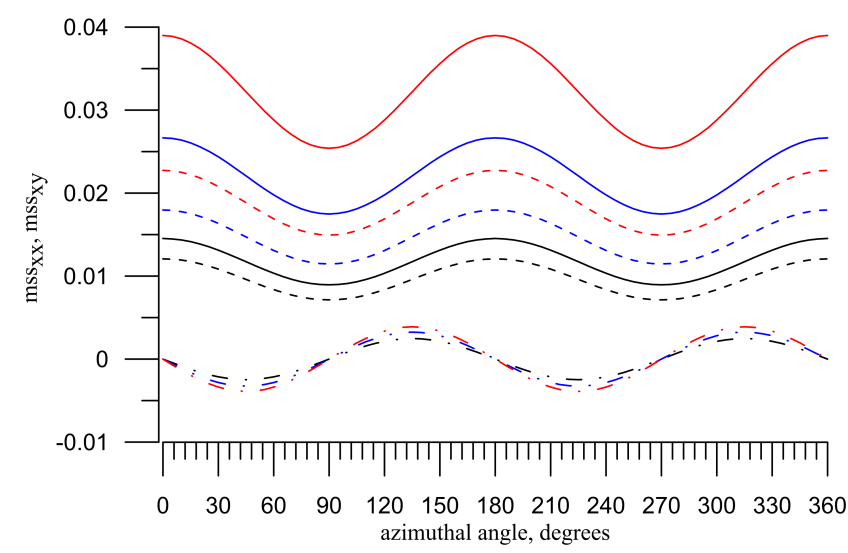

Figure 1. Azimuthal dependences of the $m s s_{x x}$ of large-scale waves (dashed line) and correlation coefficient $m s s_{x y}$ (dash-dotted line) for wind speeds of $4 \mathrm{~m} / \mathrm{s}$ (black line), $8 \mathrm{~m} / \mathrm{s}$ (blue line) and $12 \mathrm{~m} / \mathrm{s}$ (red line). Solid curves show the optical $m s s_{x x}$.

The calculations were done for a fully developed wind waves.

In Figure 1, the dashed curves show the azimuthal dependences of the $m s s_{x x}$ of large-scale waves and the dash-dotted curves show the dependences for the correlation coefficient $m s s_{x y}$ (radar wavelength $2.1 \mathrm{~cm}$ ) for three wind speeds: $4 \mathrm{~m} / \mathrm{s}$ (black curve), $8 \mathrm{~m} / \mathrm{s}$ (blue curve) and $12 \mathrm{~m} / \mathrm{s}$ (red curve). Solid curves show the dependences of the $m s s$ calculated over the entire wave spectrum, i.e. optical mss [Cox and Munk, 1954]. The azimuthal dependence for $m s s_{x x}$ is shifted by $90^{\circ}$ relative to $m s s_{y y}$ and the dependences for $m s s_{y y}$ are not shown in the figure so as not to clutter it up.

It can be seen from the figure that the mss of large-scale waves is less than the $m s s$ in the optical range. With an increase in wind speed, the $m s s$ of large-scale wave grows more slowly than optical slopes.

\section{Dependence of the NRCS on the Incidence Angle}

To calculate the dependence of the NRCS on the incidence angle, we use the full formula (2) and the statistical parameters of large-scale waves calculated in the previous section.

Figure 2 shows the dependences of the NRCS on the incidence angle for a wind speed of $4 \mathrm{~m} / \mathrm{s}$

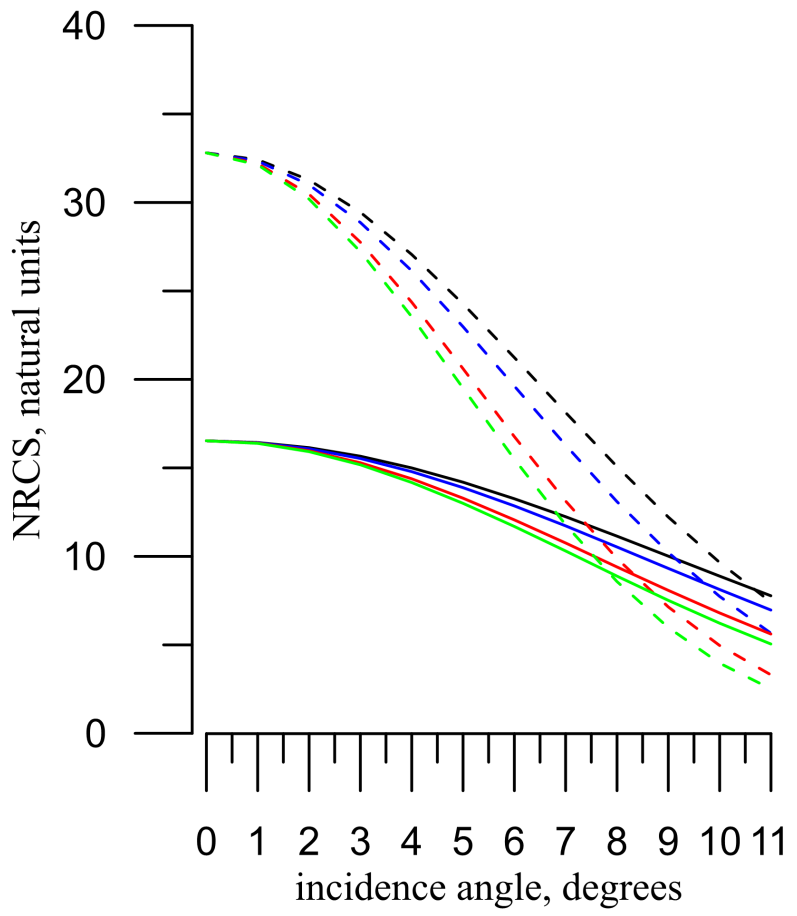

Figure 2. Dependence of the NRCS on the incidence angle for a wind speed of $4 \mathrm{~m} / \mathrm{s}$ (dashed curve) and $12 \mathrm{~m} / \mathrm{s}$ (solid curve) for different azimuthal angles: black curve $-0^{\circ}$, blue curve $-30^{\circ}$, red curve $-60^{\circ}$, and the green curve is $90^{\circ}$.

(dashed curve) and $12 \mathrm{~m} / \mathrm{s}$ (solid curve) for different azimuthal angles: black curve $-0^{\circ}$, blue curve $-30^{\circ}$, red curve $-60^{\circ}$ and the green curve is $90^{\circ}$.

It can be seen from the figure that the form of the angular dependence is determined by the $\mathrm{mss}$ of large-scale waves along the sounding direction, i.e. the greater the $m s s$, the slower the NRCS reduces with increasing the incidence angle. In our case, sounding is performed along the $X$ axis and the azimuthal angle coincides with the direction of wave propagation.

If we use formula (1), then the retrieved $\mathrm{mss}$ will lie in the range from $2 m s s_{x x}$ to $2 m s s_{y y}$, i.e. determined with a large error. This problem was solved in the developed algorithms, for example, [Karaev et al., 2019, Mitnik et al., 2020, Panfilova et al., 2020a, which retrieve the total mean square slopes of large-scale waves

$$
m s s_{\text {total }}=m s s_{x x}+m s s_{y y} .
$$

To retrieve the two-dimensional slope field of large-scale waves, it is necessary to measure at the several azimuthal angles. 


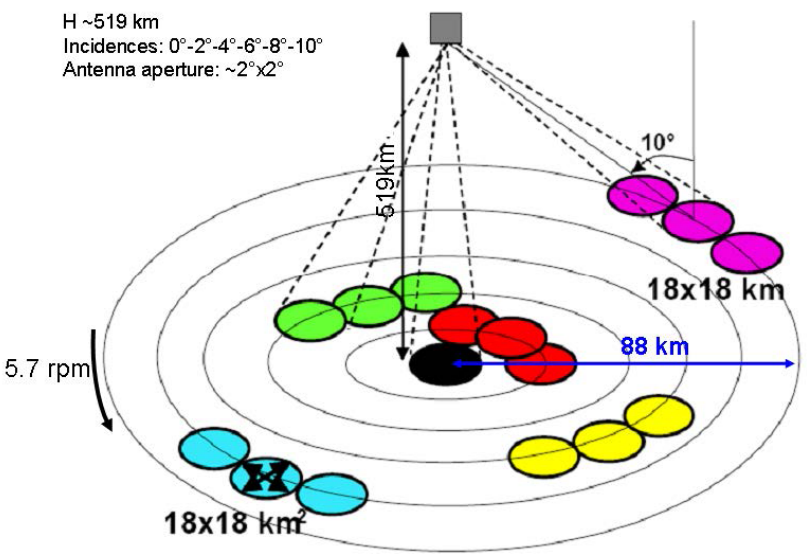

a

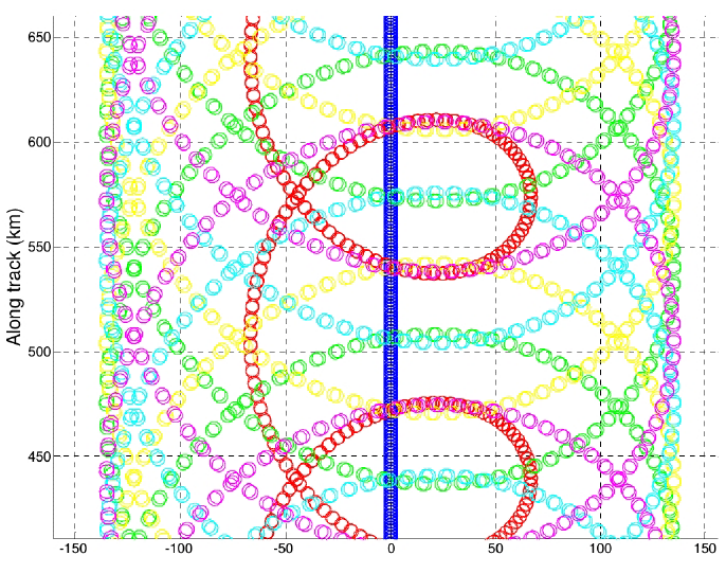

b

Figure 3. Scheme of measurements of the SWIM spectrometer (a) and the geometry of the movement of rays along the earth's surface during the movement of the CFOSAT satellite (b). Measurements are made at angles of $0^{\circ}, 2^{\circ}, 4^{\circ}, 6^{\circ}, 8^{\circ}$ and $10^{\circ}$ and a beam width of $2^{\circ}$.

\section{Spectrometer SWIM}

The concept of an orbital radar capable of measuring the dependence of the NRCS on the incidence angle at different azimuthal angles was proposed in [Karaev et al., 2006, 2010]. Two approaches were considered: rotation of the antenna system around the vertical axis and scanning mode. The latter option was chosen to be installed in the channel of near nadir sounding of the first Russian scatterometer SKAT-MP on the Meteor-MP meteorological satellite. In the new Federal Space Program (2016-2024), the satellite changed its name to Ocean and changed its focus - it became oceanological.

In recent years, remote sensing of the sea surface at small incidence angles has been actively developing, and the Chinese-French satellite CFOSAT with a SWIM spectrometer on board was launched into orbit. The measurement scheme is shown in Figure 3 a. When the satellite moves, the rays rotate and the trajectory of their movement along the underlying surface is shown in Figure 3 b.

It can be seen from the figure that due to the rotation of the antenna system around the vertical axis, the dependence of the NRCS on the incidence angle is measured at different azimuthal angles.

Let us consider an algorithm for retrieval of two-dimensional slopes from SWIM measurements, which are performed at different azimuthal angles.

\section{Retrieval Algorithm}

When for a given sea area there are measurements at several azimuthal angles, the problems of formula (1) become obvious, since for this sea area, in each azimuthal angle, its own mss of large-scale waves will be calculated and they will be different (depending on the azimuthal angle).

To apply formula (2), it is required to measure the dependence of the NRCS on the incidence angle at several azimuthal angles. The number of required azimuth angles depends on the assumptions used in the consideration.

In the case of wind waves, it is necessary to measure at 3 azimuthal angles, which will allow calculating the $\mathrm{mss}$ of the large-scale wave slopes in each azimuthal direction and find the $m s s_{\text {total }}$ of the large-scale wave slopes, the correlation coefficient and the direction of wave propagation. To do this, it is necessary to solve the following system of 3 equations

$$
\begin{gathered}
b\left(\varphi_{1}\right)= \\
\frac{m s s_{y y}\left(\varphi_{1}\right)}{2\left(m s s_{x x}\left(\varphi_{1}\right) m s s_{y y}\left(\varphi_{1}\right)-m s s_{x y}^{2}\left(\varphi_{1}\right)\right)}=b_{1}, \\
b\left(\varphi_{2}\right)= \\
\frac{m s s_{y y}\left(\varphi_{2}\right)}{2\left(m s s_{x x}\left(\varphi_{2}\right) m s s_{y y}\left(\varphi_{2}\right)-m s s_{x y}^{2}\left(\varphi_{2}\right)\right)}=b_{2},
\end{gathered}
$$




$$
\begin{gathered}
b\left(\varphi_{3}\right)= \\
\frac{m s s_{y y}\left(\varphi_{3}\right)}{2\left(m s s_{x x}\left(\varphi_{3}\right) m s s_{y y}\left(\varphi_{3}\right)-m s s_{x y}^{2}\left(\varphi_{3}\right)\right)}=b_{3},
\end{gathered}
$$

where $\varphi_{1}, \varphi_{2}, \varphi_{3}$ are azimuthal angles under which the sounding was carried out. Coefficients are calculated for each azimuthal angle using formula (4).

The situation is somewhat simplified by the fact that there is a relationship between the slopes and the correlation coefficient, which does not depend on the sounding direction

$$
m s s_{x x} m s s_{y y}-m s s_{x y}^{2}=\text { const }=A .
$$

For a wind waves propagating at an angle $\varphi_{0}$, the formulas for the $m s s_{x x}$ along the sounding directions $\varphi_{1}, \varphi_{2}, \varphi_{3}$ take the following form

$$
\begin{gathered}
m s s_{x x}\left(\varphi_{1}\right)= \\
0.5 m s s_{\text {total }}+0.5 \Delta m s s \cos \left(2 \varphi_{0}-2 \varphi_{1}\right), \\
m s s_{x x}\left(\varphi_{2}\right)= \\
0.5 m s s_{\text {total }}+0.5 \Delta m s s \cos \left(2 \varphi_{0}-2 \varphi_{2}\right), \\
m s s_{x x}\left(\varphi_{3}\right)= \\
0.5 m s s_{\text {total }}+0.5 \Delta m s s \cos \left(2 \varphi_{0}-2 \varphi_{3}\right) .
\end{gathered}
$$

In the course of further transformations, it is necessary to find 3 unknown parameters: $m s s_{\text {total }}$ of large-scale waves, the amplitude of the change of the slopes $\Delta=m s s_{x x}(\max )-m s s_{y y}(\min )$, and the direction of wave propagation. The $m s s_{x x}\left(\varphi_{n}\right)$ along any direction $\varphi_{n}$ can then be easily calculated.

After the standard transformations in solving the system of equations (12)-(14), we obtain the following formulas

$$
\begin{gathered}
\operatorname{tg}\left(2 \varphi_{0}\right)=\left[\left(b_{2}-b_{1}\right) \sin \left(\varphi_{1}+\varphi_{3}\right) \sin \left(\varphi_{1}-\varphi_{3}\right)-\right. \\
\left.\left(b_{3}-b_{1}\right) \sin \left(\varphi_{1}+\varphi_{2}\right) \sin \left(\varphi_{1}-\varphi_{2}\right)\right] / \\
{\left[\left(b_{2}-b_{1}\right) \cos \left(\varphi_{1}+\varphi_{3}\right) \sin \left(\varphi_{1}-\varphi_{3}\right)-\right.} \\
\left.\left(b_{3}-b_{1}\right) \cos \left(\varphi_{1}+\varphi_{2}\right) \sin \left(\varphi_{1}-\varphi_{2}\right)\right]
\end{gathered}
$$

$$
\begin{gathered}
\Delta m s s=A \times \\
\frac{4\left(b_{2}-4 b_{1}\right)}{\cos \left(2 \varphi_{0}-2 \varphi_{1}\right)-\cos \left(2 \varphi_{0}-2 \varphi_{2}\right)}, \\
m s s_{\text {total }}=A \times \\
{\left[4 b_{1}+\frac{4\left(b_{2}-b_{1}\right) \cos \left(2 \varphi_{0}-2 \varphi_{1}\right)}{\cos \left(2 \varphi_{0}-2 \varphi_{1}\right)-\cos \left(2 \varphi_{0}-2 \varphi_{2}\right)}\right] .}
\end{gathered}
$$

The direction of wave propagation is determined by formula 19 , which will allow us to move forward in the solution. The parameter that was defined above remains unknown. The resulting system of equations (20)-21) cannot be solved explicitly and it is necessary to use methods for solving transcendental equations for the following expression:

$$
\begin{gathered}
m s s_{x x}+m s s_{y y}-\left(m s s_{x x} m s s_{y y}-m s s_{x y}^{2}\right) \times \\
{\left[4 b_{1}+\frac{4\left(b_{2}-b_{1}\right) \cos \left(2 \varphi_{0}-2 \varphi_{1}\right)}{\cos \left(2 \varphi_{0}-2 \varphi_{1}\right)-\cos \left(2 \varphi_{0}-2 \varphi_{2}\right)}\right]} \\
=0
\end{gathered}
$$

where to calculate the wave parameters, formulas (7) - (9) are used and the variables are the wind speed at a height of $10 \mathrm{~m} U_{10}$ and the dimensionless length of the wind fetch $\tilde{x}$, which describe the wave spectrum [Ryabkova et al., 2019].

However, the solution can be obtained in an explicit form if we assume that the coefficient $m s s_{x y}$ is much less than the mss of large-scale waves and neglect it in formula (5). Figure 1 shows that this condition is satisfied quite well.

Then the basis for further transformations will be formula (5) written under the assumption that $m s s_{x y}=0$

$$
B_{n}=\frac{1}{2 b_{n}} .
$$

As a result, the solution to a system of three equations similar to (12)-14 has the following form

$$
\begin{gathered}
\varphi_{0}= \\
0.5 \operatorname{arctg}\left\{\left[\left(B_{2}-B_{1}\right) \sin \left(\varphi_{3}-\varphi_{1}\right) \sin \left(\varphi_{1}+\varphi_{3}\right)\right.\right. \\
\left.-\left(B_{3}-B_{1}\right) \sin \left(\varphi_{2}-\varphi_{1}\right) \sin \left(\varphi_{1}+\varphi_{2}\right)\right] /
\end{gathered}
$$




$$
\begin{gathered}
{\left[\left(B_{2}-B_{1}\right) \sin \left(\varphi_{3}-\varphi_{1}\right) \cos \left(\varphi_{1}+\varphi_{3}\right)-\right.} \\
\left.\left.\left(B_{3}-B_{1}\right) \sin \left(\varphi_{2}-\varphi_{1}\right) \cos \left(\varphi_{1}+\varphi_{2}\right)\right]\right\} \\
\Delta m s s= \\
\frac{2\left(B_{2}-B_{1}\right)}{\cos \left(2 \varphi_{0}-2 \varphi_{2}\right)-\cos \left(2 \varphi_{0}-2 \varphi_{1}\right)}, \\
2 B_{1}-\frac{2\left(B_{2}-B_{1}\right) \cos \left(2 \varphi_{0}-2 \varphi_{1}\right)}{\cos \left(2 \varphi_{0}-2 \varphi_{2}\right)-\cos \left(2 \varphi_{0}-2 \varphi_{1}\right)}
\end{gathered}
$$

The obtained values can be used to estimate the interval of variation of the parameters when solving the transcendental equation 22).

In Figure 4, the dotted line shows the azimuthal dependence of the retrieved $m s s_{x x}$ of large-scale waves according to the simplified version (formulas (24) - 26p) and the solid curve shows the true value of the $m s s_{x x}$ for three wind speeds: black curve $-4 \mathrm{~m} / \mathrm{s}$, blue curve $-8 \mathrm{~m} / \mathrm{s}$ and the red curve is $12 \mathrm{~m} / \mathrm{s}$. It can be seen from the figure that with increasing wind speed the absolute value of the error grows and the maximum value of the error reaches at azimuthal angles of $45^{\circ}, 135^{\circ}, 225^{\circ}$ and $315^{\circ}$. The solution is accurate when sounding along or across directions of wave propagation.

The SWIM spectrometer performs measurements at different azimuthal angles to retrieve a twodimensional large-scale wave slope field. As an additional parameter according to the "reconstructed" wave spectrum, estimates of the significant wave height can be obtained, which can be compared with the measurements of the altimeter, which is part of the SWIM radar complex. A similar approach was used in [Panfilova et al., 2020b].

\section{Example of the Algorithm Application}

Processing and analyzing SWIM data, which will allow to check the performance of the proposed algorithm and evaluate its accuracy, is a separate large research and is beyond the scope of this paper. However, to illustrate the operation of the algorithm, we will give the example of its use.

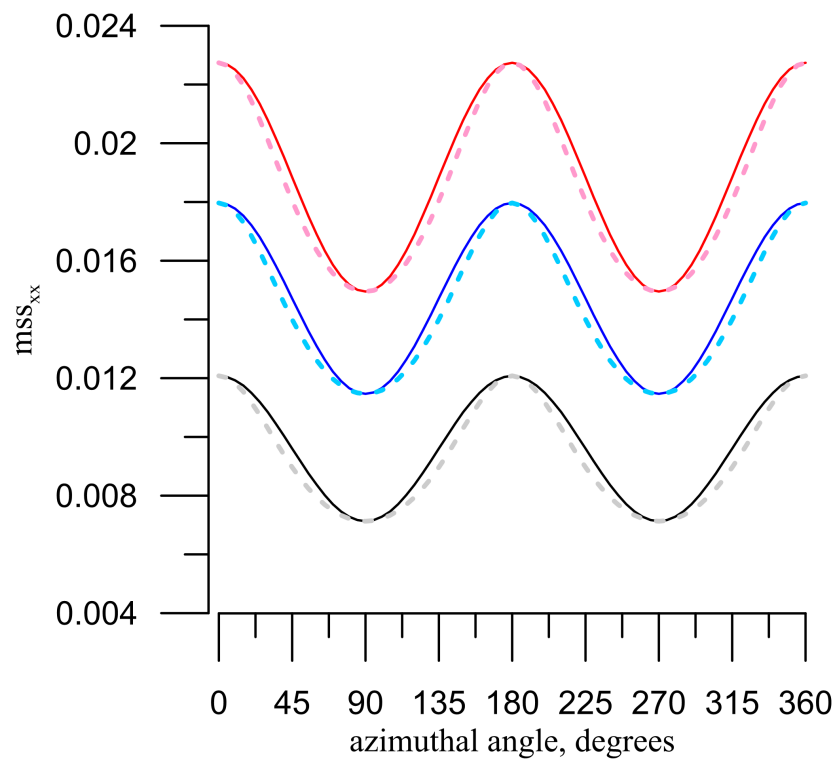

Figure 4. Azimuthal dependence of the of large-scale waves: solid curve - model value, dotted line - retrieved using a simplified formula. The calculations were performed for a wind speed of $4 \mathrm{~m} / \mathrm{s}$ (black curves), $8 \mathrm{~m} / \mathrm{s}$ (blue curves), and $12 \mathrm{~m} / \mathrm{s}$ (red curves).

In Figure 5 asterisks show the result of the wellknown algorithm for retrieval of slopes of largescale waves from the dependence of the NRCS on the incidenvce angle. These algorithms have been used to process precipitation radar data from TRMM and GPM satellites, for example [Chu et al., 2012a Freilich and Vanhoff, 2003; Panfilova et al., 2020a.

The scatter of the retrieved values observed in the figure will lead to an error in determining the slopes and direction of wave propagation when using only three azimuth angles.

To reduce the error, it is proposed to use two approaches: 1) it is necessary that among the three selected azimuthal angles, the maximum difference between the angles is not less than $90^{\circ} ; 2$ ) the obtained estimates of the slopes are averaged over a given set of triplets of azimuthal angles.

The result of this approach is shown in Figure 5 blue curve. The azimuthal dependence of the is given by the formula (16), where $m s s_{\text {total }}=$ $0.03194, \Delta m s s=0.002$ and $\varphi_{0}=-6.3^{\circ}$.

Good agreement is seen between the model dependence and the measured data, which confirms the efficiency of the developed theoretical algorithm. 


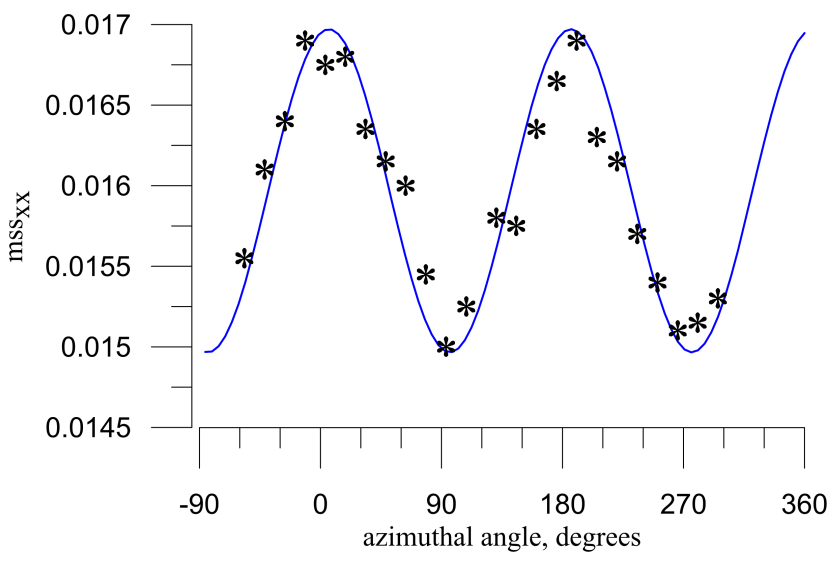

Figure 5. Azimuthal dependence of the of large-scale waves: asterisks - retrieved values and a blue curve - model.

\section{Conclsuion}

The orbiting of the SWIM spectrometer installed on the Chinese-French satellite CFOSAT opens up the possibility of measuring a two-dimensional large-scale wave slope field for the first time. Measurements of the NRCS are performed at small incidence angles $\left(<11^{\circ}\right)$ at different azimuthal angles, when the quasi-specular backscattering mechanism is dominant. At small incidence angles, the mss of large-scale wave slopes is retrieved along the probing direction. Such an algorithm was developed for precipitation radars installed on the TRMM and GPM satellites of the JAXA. However, the measurements were carried out at one azimuthal angle, therefore, an original data analysis method was proposed that allows one to determine the total mss of large-scale waves [Karaev et al., 2019 . Panfilova et al., 2020a.

In the SWIM spectrometer, measurements are performed at different azimuthal angles, which makes it possible to determine the direction of propagation of the dominant wave system, measure the $m s s$ along the sounding direction, and for the first time retrieve a two-dimensional field of large-scale wave slopes.

Numerical modeling was carried out and the dependences of the NRCS on the incidence angle were plotted for different azimuthal angles. A processing algorithm is proposed that uses measurements at three azimuthal angles and requires, in the general case, the solution of the transcendental equation. If we neglect the correlation coefficient, then the solu- tion is in analytical form. Estimates of the error in reconstructing the slopes of large-scale waves by a simplified algorithm in comparison with the exact solution are obtained. An example of SWIM data processing is given.

The developed algorithms will make it possible to retrieve a two-dimensional field of large-scale slopes using data from the SWIM spectrometer and to analyze processes on the sea surface.

Acknowledgments. This work was funded by the Russian Science Foundation (Project RSF 20-17-00179).

\section{References}

Barrick, D. E. (1968), Rough surface scattering based on the specular point theory, IEEE Transactions Antenna and Propagation, 16, No. 4, 449-554, Crossref

Bass, F. G., I. M. Fuks (1972), Wave Scattering on a Statistically Rough Surface, 424 pp. Nauka, Moscow. (in Russian)

Birol, F., E. Blayo, J. M. Brankart, et al. (2002), Advanced Altimeter Data Assimilation for the Development of Operational Oceanography, INSU, France. (https://www.aviso.altimetry.fr/fileadmin/document; /OSTST/2002_b/verron.pdf)

Boisot, O., F. Nouguier, et al. (2015), The GO4 Model in Near-Nadir Microwave Scattering From the Sea Surface, IEEE Transactions on Geoscience and Remote Sensing, 53, No. 11, 5889-5900, Crossref

Chen, P., G. Zheng, et al. (2017), Quasi-Gaussian probability density function of sea wave slopes from near nadir Ku-band radar observations, Remote Sensing of Environment, 217, 86-100, Crossref

Chu, X., Y. He, et al. (2012a), Relationships between $\mathrm{Ku}$-band radar backscatter and integrated wind and waves parameters at low incidence angles, IEEE Transactions on Geoscience and Remote Sensing, 50, No. 11, 4599-4609, Crossref

Chu, X., Y. He, G. Chen (2012b), Asymmetry and anisotropy of microwave backscatter at low incidence angles, IEEE Transactions on Geoscience and Remote Sensing, 50, No. 10, 4014-4024, Crossref

Cox, C., W. Munk (1954), Measurements of the roughness of the sea surface from photographs of the sun glitter, J. Opt. Soc. Amer., 44, No. 11, 838-850, Crossref

Danilytchev, M., B. Kutuza, A. Nikolaev (2009), The application of sea wave slope distribution empirical dependencies in estimatioin of interaction between microwave radiation and rough sea surface, IEEE Transactions on Geoscience and Remote Sensing, 47, No. 2, 652-661, Crossref

Freilich, M. H., B. A. Vanhoff (2003), The relation between winds, surface roughness, and radar 
backscatter at low incidence angles from TRMM Precipitation Radar measurements, Journal of Atmospheric and Oceanic Technology, 20, No. 4, 549562, Crossref

Garnakeryan, A. A., $\quad$ A. S. Sosunov (1978), $\quad R a-$ diolocation of the Sea Surface, 144 pp. Izdatel'stvo Rostovskogo Universiteta, Rostov. (in Russian)

GPM (2014), GPM Data Utilization Handbook. First Edition, 92 pp. JAXA, Tokyo.

Isakovich, M. A. (1952), Scattering of waves from a statistically rough surface, Zhurnal Ehksperimental'noi i Teoreticheskoi Fiziki, 23, No. 3, 305-314. (in Russian)

Karaev, V., M. Kanevsky, et al. (2006), A rotating knife-beam altimeter for wide-swath remote sensing of the ocean: wind and waves, Sensors, 6, 260-281, Crossref

Karaev, V., M. Kanevsky, et al. (2010), The concept of the advanced space microwave radar for remote sensing of the ocean at small incidence angles, PORSEC 2010 Proceedings p. 23, PORSEC, Taiwan.

Karaev, V., M. Panfilova, et al. (2012), Retrieval of the variance of the slopes of large-scale waves from radar measurements in the microwave range, Issledovanie Zemli iz Kosmosa, No. 4, 62-77. (in Russian)

Karaev, V., M. Panfilova, et al. (2018), Experiment at the International Space Station: a microwave radar with scanning fan beam antenna at nadir probing, 25 years of Progress in Radar Altimetry Symposium, 2018, Abstracts p. 232-233, ESA, Portugal.

Karaev, V., L. Mitnik, et al. (2019), Mean square slopes of sea waves in cyclone area from dualfrequency precipitation radar and microwave radiometer, Proc. of IGARSS 2019 p. 8109-8112, IGARSS, Crossref

Martina, M. J., M. Balmasedab, L. Bertinoc, et al. (2015), Status and future of data assimilation in operational oceanography, Journal of Operational Oceanography, 8, No. S1, s28-s48, Crossref

Mitnik, L., V. Kuleshov, et al. (2020), Retrieval of mean square slopes of sea waves, surface wind speed, total water vapor content and total cloud liquid water content in Hagibis typhoon area from satellite active and passive microwave, International Geoscience and Remote Sensing Symposium (IGARSS 2020), Proceedings p. 5709-5712, IGARSS, Crossref

Nouguier, F., A. Mouche, et al. (2014), Analysis of dual-frequency ocean backscatter measurements at $\mathrm{Ku}-$ and Ka-band using near-nadir incidence GPM radar data, Journal of Latex Class Files, 13, No. 9, $1-5$, Crossref
Nouguier, F., A. Mouche, et al. (2016), Analysis of Dual-Frequency Ocean Backscatter Measurements at Ku- and Ka-Bands Using Near-Nadir Incidence GPM Radar Data, IEEE Geoscience and Remote Sensing Letters, 13, No. 9, 1310-1314, Crossref

Panfilova, M., V. Karaev, J. Guo (2018), Oil slick observation at low incidence angles in Ku-band, Journal of Geophysical Research, Oceans, 123, No. 3, 1924-1936, Crossref

Panfilova, M., V. Karaev, et al. (2020a), Advanced view at the Ocean Surface, Journal of Geophysical Research, Oceans, 125, No. 11, Crossref

Panfilova, M., M. Ryabkova, et al. (2020b), Retrieval of the statistical characteristics of wind waves from the width and shift of the Doppler spectrum of the backscattered microwave signal at low incidence angles, IEEE Transactions on Geoscience and Remote Sensing, 20, No. 3, 2225-2231, Crossref

Pokazeev, K. V., A. S. Zapevalov (2019), Determining higher-order cumulants of sea-surface slopes by quasi-vertical radio sounding data, Vestnik Moskovskogo Universiteta, Seriya 3: Fizika, Astronomiya, 3, 73-76, Crossref

Ryabkova, M., V. Karaev, et al. (2019), A review of wave spectra models as applied to the problem of radar probing of the sea surface, Journal of Geophysical Research, Oceans, 124, 1-31, Crossref

Tran, N., B. Chapron, D. Vandemark (2007), Effects of long waves on Ku-band ocean radar backscatter at low incidence angles using TRMM and altimeter data, IEEE Transactions on Geoscience and Remote Sensing, 4, No. 4, 542-546, Crossref

TRMM (2001), TRMM Data Users Handbook (2001), 226 pp. NASDA, Tokyo.

Valenzuela, G. (1978), Theories for the interaction of electromagnetic and oceanic waves - A review, Boundary Layer Meteorology, 13, 61-86, Crossref Yan, Q., J. Zhang, et al. (2019), Analysis of Kuand Ka-Band Sea Surface Backscattering Characteristics at Low-Incidence Angles Based on the GPM Dual-Frequency Precipitation Radar Measurements, Remote Sensing, 11,754, Crossref

Zapevalov, A. S. (2020), Distribution of the variance of the sea surface slopes over the spatial ranges of the waves that create them, Sovremennye Problemy Distantsionnogo Zondirovaniya Zemli iz Kosmosa, 17, No. 1, 211-219, (in Russian)Crossref

\section{Corresponding author:}

V. Yu. Karaev, Institute of Applied Physics RAS, 603950, Nihzny Novgorod. (volody@ipfran.ru) 